\title{
Mutation analysis of the phenylalanine hydroxylase gene and its clinical implications in two Japanese patients with non-phenylketonuria hyperphenylalaninemia
}

\begin{abstract}
We describe a mutation analysis for the phenylalanine hydroxylase gene and the clinical outcome of two Japanese patients with non-phenylketonuria (PKU) hyperphenylalaninemia (serum phenylalanine level below $600 \mu \mathrm{mol} / 1$ under a free diet) detected by a mass-screening program. Single strand conformation polynorphism analysis and direct sequencing of their genomic DNAs revealed that non-PKU hyperphenylalaninemia resulted from compound heterozygosity for a mutation causing classical PKU and a mutation with a milder effect on phenylalanine hydroxylase activity. The mutations were R241C and R243Q in exon 7, and R413P in exon 12. The mutation genotypes of the two patients were R241C/R243Q and R241C/R413P. It has been demonstrated that homozygosity for the R243Q or R413P mutation is associated with a severe phenotype of PKU and low in vitro expression activity. In contrast, the R241C mutation has much less effect on phenylalanine hydroxylase activity. The metabolic consequence of each variant allele was confirmed by a phenylalanine loading test in the patients and their parents. The patients achieved normal results in all intellectual and neurologic tests. Brain magnetic resonance imaging revealed no abnormalities. The dietary restriction was continued until 10 years of age in order to maintain the serum phenylalanine level below $400 \mu \mathrm{mol} / \mathrm{l}$. The genetic analysis to distinguish non-PKU hyperphenylalaninemia from classical PKU helps to determine the principles of dietary management in the early infantile period.
\end{abstract}

Key words Phenylketonuria (PKU) · Non-phenylketonuria hyperphenylalaninemia · Phenylalanine hydroxylase $(\mathrm{PAH}) \cdot$ Missense mutation $\cdot$ Compound heterozygote

M. Kibayashi $\cdot$ M. Nagao $(\square)$

Department of Pediatrics, National Otaru Hospital, 3-24-1

Nagahashi, Otaru 047-0036, Japan

Tel. +81-134-32-5131, Fax +81-134-29-2164

e-mail: CXQ04341@niftyserve.or.jp

M. Kibayashi $\cdot$ M. Nagao $\cdot$ S. Chiba

Department of Pediatrics, Sapporo Medical University, Sapporo,

Japan

\section{Introduction}

Phenylketonuria (PKU) is an autosomal recessive inherited metabolic disorder caused by a deficiency of hepatic phenylalanine hydroxylase (PAH) (MIM 261600). A mass screening program for neonatal hyperphenylalaninemia was started in Japan about 20 years ago, and classical PKU was observed in 1 in 120,000 births (Aoki and Wada 1988). In the course of the program, it became clear that PKU is phenotypically heterogeneous, ranging from a moderate elevation of plasma phenylalanine levels without neurological impairment to classical PKU (Güttler 1980). The classification of hyperphenylalaninemia is made by measurement of serum phenylalanine levels under a free diet: levels above $1200 \mu \mathrm{mol} / \mathrm{l}$ indicate classical PKU; levels between 600 and $1200 \mu \mathrm{mol} / 1$, mild PKU; and below $600 \mu \mathrm{mol} / \mathrm{l}$, nonPKU hyperphenylalaninemia. The heterogeneity of clinical phenotypes can be explained by residual PAH activity in the liver. It was recognized that patients with non-PKU hyperphenylalaninemia appear to show normal intellectual and behavioral development without treatment (Levy et al. 1971). However, Costello et al. (1994) reported that untreated patients with mild PKU and non-PKU hyperphenylalaninemia presented a lower IQ compared with treated patients. Therefore, a lifelong diet to maintain serum phenylalanine levels below $400 \mu \mathrm{mol} / 1$ was recommended. Also, the precise hyperphenylalaninemic phenotype is not always evident on the basis of the degree of hyperphenylalaninemia immediately after the mass screening. A follow-up of patients with phenylalanine restriction is sometimes necessary to classify the phenotype. The confusion in diagnosis of the PKU phenotype complicates an appropriate follow-up and dietary management in childhood.

Technical progress in molecular genetics has made it possible to detect directly the molecular defect in DNA associated with PKU. Presently, more than 20PKU mutations among Japanese and other Oriental populations have been reported (Wang et al. 1989, 1991a, 1991b, 1991c, 1992; Lin et al. 1992; Okano et al. 1994a, 1994b; Takarada et al. 
1994). The heterogeneity of clinical phenotypes in European patients can be explained by a multiplicity of mutations in the PAH gene (Okano et al. 1991). However, the molecular basis for the phenotypic diversity among Japanese PKU patients is not clear. It is important to know whether non-PKU hyperphenylalaninemia is associated with classical PKU alleles or unknown variant alleles.

The present study demonstrates that in two families, patients were compound heterozygotes of PKU alleles, one of which has a milder effect on PAH activity. The clinical consequences of this disorder, including results of intellectual and neurologic tests, are also described.

\section{Materials and methods}

\section{Patients}

Patient A (currently 15 years of age) is the second child of healthy, unrelated parents of Japanese origin. The family history, pregnancy, and birth, at term, were uneventful. Hyperphenylalaninemia $(488 \mu \mathrm{mol} / \mathrm{l})$ was detected by a mass-screening program in Hokkaido, Japan. Urinary excretion of biopterin and neopterin, and the neopterin/ biopterin ratio, were normal. Dihydropteridine reductase activities in erythrocytes were within the normal range. Measurement of the dietary intake of protein and mild restriction of phenylalanine were continued until 10 years of age, with a diagnosis of mild PKU or non-PKU hyperphenylalaninemia. The patient was finally diagnosed with non-PKU hyperphenylalaninemia from the results of the phenylalanine tolerance test at 10 years of age. Phenylalanine tolerance was determined by the amount of phenylalanine $(\mathrm{mg} / \mathrm{kg}$ body weight per day) in order to keep the serum phenylalanine levels below $600 \mu \mathrm{mol} / 1$. His serum phenylalanine values have been less than $600 \mu \mathrm{mol} / 1$ on a normal diet.

Patient B (currently 13 years of age) is the first child of Japanese parents who are unrelated. The pregnancy, birth, and family history were uneventful. Hyperphenylalaninemia $(542 \mu \mathrm{mol} / \mathrm{l})$ was detected by the neonatal screening program. Defects in biopterin metabolism were excluded. Dietary treatment was continued until 10 years of age, and the mean levels of serum phenylalanine were well controlled below $400 \mu \mathrm{mol} / \mathrm{l}$. He was diagnosed as having nonPKU hyperphenylalaninemia by the assessment of a daily phenylalanine tolerance test.

\section{Oral loading tests of L-phenylalanine}

The oral loading tests of L-phenylalanine $(100 \mathrm{mg} / \mathrm{kg}$ body weight) was carried out in each patient at 10 years of age and in their parents. After an overnight fast, L-phenylalanine (dissolved in $0.01 \mathrm{~N} \mathrm{HCl}$ ) was administered to the subjects at 7 a.m. Blood samples were obtained before and at 1, 2, 4, 24, and $48 \mathrm{~h}$ after the load. The plasma phenylalanine and tyrosine concentrations were measured at each time. Informed consent was obtained for all subjects examined.

\section{Mutation analysis}

The genomic DNA samples were prepared from peripheral blood leukocytes by the method previously described (Gustafson et al. 1987). The coding sequence and flanking region of each exon were scanned for mutations by polymerase chain reaction (PCR)-single strand conformation polymorphism. The nucleotide sequence of each set of primers was described previously (Wang et al. 1989, 1991a, 1991b, 1991c, 1992; Lin et al. 1992; Takarada et al. 1994). The exonic regions containing a variant sequence were subjected to direct sequencing analysis. The patients and their family members were characterized with respect to mutation genotype in the PAH gene.

\section{Results}

Mutation analysis

Preliminary restriction fragment length polymorphism (RFLP) analysis revealed that haplotype 4 comprised $100 \%$ of the variant alleles examined in this study. We then amplified each exon and its flanking intronic regions of the $\mathrm{PAH}$ gene, and scanned for mutations in genomic DNA from the patients and their parents. Two different mutations were characterized in each family. The results of sequence analysis and segregation of each variant allele in two families are illustrated in Fig. 1. In patient A, the R241C (a C-to-T transition at the first base of codon 241) and the R243Q (a G-to-A transition at the second base of codon 243) mutations were detected in exon 7 . The carrier status of the parents was also determined by direct sequencing of exon 7 . Patient B was heterozygous for the R241C mutation in exon 7 and the R413P (a G-to-C transition at the second base of codon 413) mutation in exon 12. It was determined that the R413P allele originated from the father and the R241C allele from the mother by sequencing exons 7 and 12 of the parents.

\section{Phenylalanine loading tests}

Phenylalanine loading tests were performed to evaluate the in vivo consequence of heterozygosity for one of these mutations (Fig. 2). The maximum concentrations of serum phenylalanine were reached at 1 or $2 \mathrm{~h}$ after the load. Although the serum concentrations before the load were higher in the patients than in their parents and in controls, the loading dose of phenylalanine was eliminated in all subjects within $24 \mathrm{~h}$. No significant difference was observed in the pattern of the phenylalanine elimination curves. The father of patient $\mathrm{A}$ and the mother of patient B, who were both heterozygous for the R241C mutation, formed more tyrosine than the parents heterozygous for R243Q or R413P. However, the two patients, who were compound heterozygotes of these mutations, did not respond with increase in tyrosine (Table 1). 
Fig. 1 Identification of missense mutations in the PAH gene by the PCR cycle sequencing method. Primers used for PCR amplification and sequencing are described by Wang et al. (1991a, 1991c). A R241C and R243Q mutations in exon 7 of patients A and B. B R413P mutation in exon 12 of patient B. C Segregation of variant alleles in the two families presenting with non-PKU hyperphenylalaninemia
A

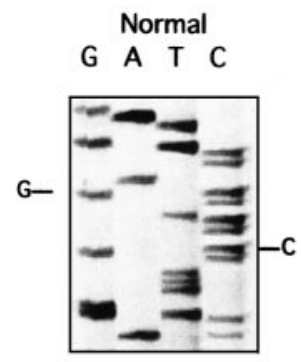
Patient A
G A T C

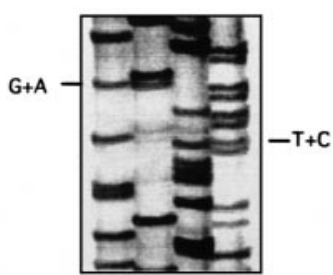

Arg 243
Normal 5'-T T T C C G C C T C C G A C C T G-3'
Patient B

G A T C

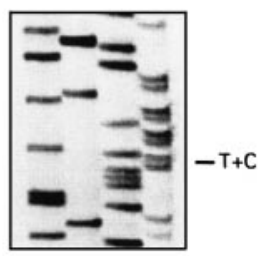

Normal

G A T C

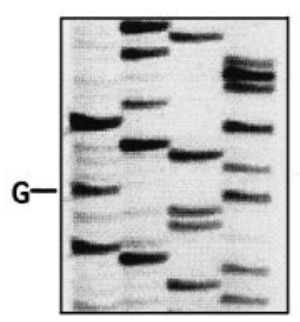

Patient B

G A T C

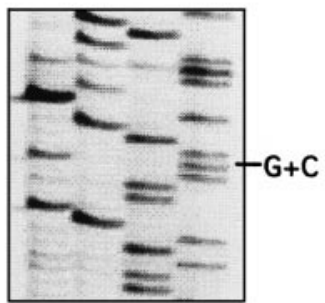

Arg 413

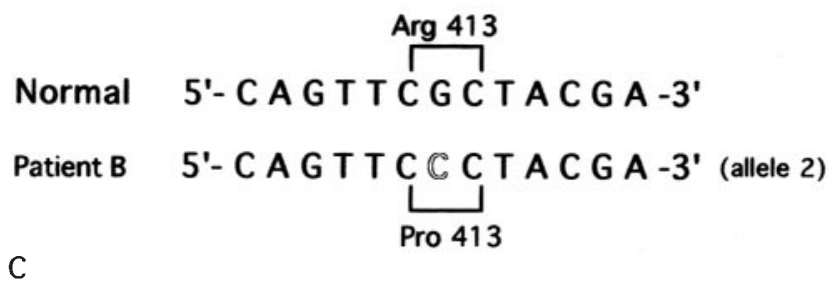

Family A

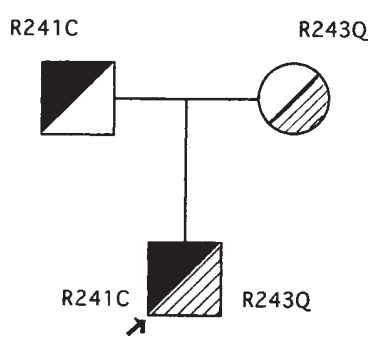

Family B

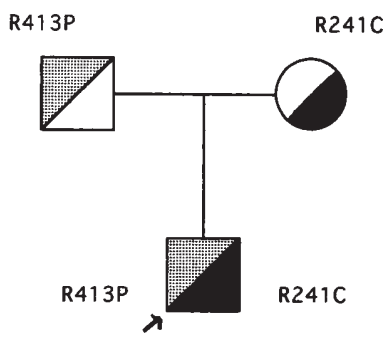


Table 1 Mutation genotypes and biochemical phenotypes of individuals in two families with non-PKU hyperphenylalaninemia

\begin{tabular}{|c|c|c|c|c|}
\hline & Genotype & $\begin{array}{l}\text { Phenylalanine } \\
\text { level }(\mu \mathrm{mol} / \mathrm{l})^{\mathrm{a}}\end{array}$ & $\begin{array}{l}\text { Plasma tyrosine response after } \\
\text { phenylalanine loading }(\mu \mathrm{mol} / 1)^{\mathrm{b}}\end{array}$ & Phenotype \\
\hline \multicolumn{5}{|l|}{ Family $A$} \\
\hline Patient A & $\mathrm{R} 241 \mathrm{C} / \mathrm{R} 243 \mathrm{Q}$ & 269 & -6 & Non-PKU HPA \\
\hline Father & R241C/wild & 48 & +112 & Carrier \\
\hline Mother & R243Q/wild & 70 & +68 & Carrier \\
\hline \multicolumn{5}{|l|}{ Family $B$} \\
\hline Patient B & $\mathrm{R} 241 \mathrm{C} / \mathrm{R} 413 \mathrm{P}$ & 387 & -8 & Non-PKU HPA \\
\hline Father & R413P/wild & 96 & +42 & Carrier \\
\hline Mother & R241C/wild & 38 & +95 & Carrier \\
\hline
\end{tabular}

PUK, phenylketonuria; HPA; hyperphenylalaninemia

${ }^{a}$ Serum phenylalanine levels under a free diet

${ }^{\mathrm{b}}$ Maximum increase $(+)$ or decrease $(-)$ in plasma tyrosine levels after the phenylalanine loading test

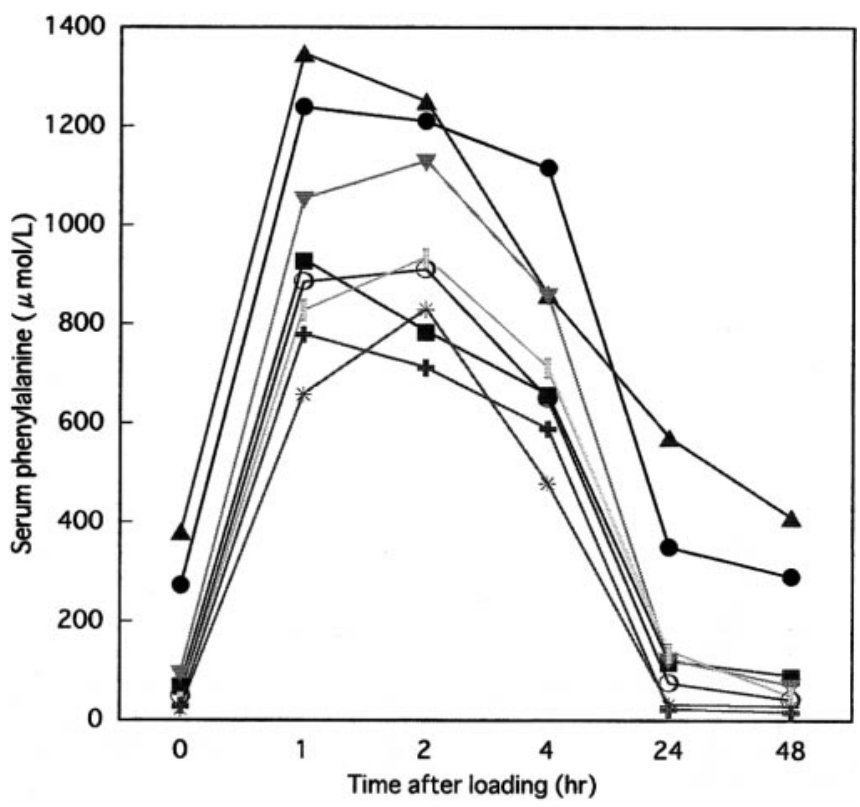

Fig. 2 Serum phenylalanine levels after L-phenylalanine loading. LPhenylalanine $(100 \mathrm{mg} / \mathrm{kg}$ body weight) was given orally after an overnight fast. Blood specimens were obtained before and at 1, 2, 4, 24, and $48 \mathrm{~h}$ after the load. Solid circle, patient A; open circle, father of patient $\mathrm{A}$; solid square, mother of patient A; solid triangle, patient $\mathrm{B}$; inverted solid triangle, father of patient $\mathrm{B}$; vertical rule, mother of patient $\mathrm{B}$, cross, control 1; asterisk, control 2

Intellectual and neurologic tests

The serum phenylalanine levels of the patients were maintained below $400 \mu \mathrm{mol} / \mathrm{l}$ by dietary restriction of phenylalanine until 10 years of age. With a definite diagnosis of non-PKU hyperphenylalaninemia as described above, dietary restriction was subsequently discontinued. The serum phenylalanine levels were less than $600 \mu \mathrm{mol} / \mathrm{l}$ on a normal dietary intake of protein. Clinical neurologic examinations, including electroencephalography (EEG), auditory brainstem response (ABR), and short-latency somatosensory evoked potential (SSEP), revealed no abnormalities in either patient. Their general, verbal, and performance IQ, as measured by the Wechsler Intelligence Scale for
Table 2 Serum phenylalanine levels and intelligence scores in patients with non-PKU hyperphenylalaninemia

\begin{tabular}{|c|c|c|}
\hline & Patient A & Patient B \\
\hline \multicolumn{3}{|l|}{$\begin{array}{l}\text { Serum phenylalanine } \\
\text { levels }(u \mathrm{~mol} / 1)^{\mathrm{a}}\end{array}$} \\
\hline Screening & 488 (breastfeeding) & 542 (breastfeeding) \\
\hline $0-1$ year $(n=12)$ & $362 \pm 118$ & $408 \pm 139$ \\
\hline $1-6$ years $(n=20)$ & $349 \pm 150$ & $375 \pm 106$ \\
\hline $7-10$ years $(n=16)$ & $288 \pm 88$ & $336 \pm 124$ \\
\hline$\geqslant 11$ years $(n=16)$ & $451 \pm 135$ & $497 \pm 108$ \\
\hline \multicolumn{3}{|c|}{$\begin{array}{l}\text { Phenylalanine restriction } \\
\text { (mg/kg body weight) }\end{array}$} \\
\hline $0-1$ year & $50-70$ & $50-70$ \\
\hline $1-6$ years & $60-80$ & $60-75$ \\
\hline $7-10$ years & $70-85$ & $65-80$ \\
\hline$\geqslant 11$ years & Free & Free \\
\hline \multicolumn{3}{|l|}{$\mathrm{IO}^{\mathrm{b}}$} \\
\hline Full-scale & 104 & 108 \\
\hline Verbal & 101 & 104 \\
\hline Performance & 106 & 110 \\
\hline Genotype & $\mathrm{R} 241 \mathrm{C} / \mathrm{R} 243 \mathrm{Q}$ & R241C/R413P \\
\hline
\end{tabular}

${ }^{a}$ Serum phenylalanine levels are indicated as mean \pm SD

${ }^{\mathrm{b}}$ Deviation IQ scores with a mean $( \pm)$ of $100 \pm 15$

Children-Revised (WISC-R), were within the normal range (Table 2). Brain magnetic resonance imaging (MRI) did not show any abnormalities in cerebral white matter or other areas. The patients are currently 15 (patient A) and 13 (patient B) years of age, and their physical growth is satisfactory.

\section{Discussion}

The molecular basis of non-PKU hyperphenylalaninemia has not been well investigated in the Japanese population, although several mutations were described in patients with classical PKU. Since RFLP analysis of the PAH gene revealed that haplotype 4 dominated in this population (Daiger et al. 1989), the application of haplotype analysis is not very informative for diagnosis. In this study, using PCRbased methods, we could identify several missense muta- 
tions by direct analysis of the PAH gene in two patients with non-PKU hyperphenylalaninemia. We have also confirmed the previous finding that the combined expression of a severe PKU allele and an allele with a milder effect on PAH activity results in non-PKU hyperphenylalaninemia (Economou-Petersen et al. 1992).

We have found an association of the R241C mutation with non-PKU hyperphenylalaninemia in two families. Okano et al. (1994b) reported that this mutation is uncommon, the frequency being $5 \%$ of all PKU alleles identified so far in Japan. They revealed that the PAH activity of the mutant protein was $25 \% \pm 3 \%$ (SD) of normal (Okano et al. 1994a). We identified another two mutations (R243Q and R413P) previously described in classical PKU patients. The R243Q mutation accounts for $7.9 \%$ of all PKU chromosomes in the Japanese population (Okano et al. 1994b). The R243Q mutation produced less than $10 \%$ of normal PAH activity in in vitro expression, and patients homozygous for the substitution exhibit a severe clinical phenotype (Wang et al. 1991c). The R413P mutation is maintained at a relatively high frequency (around 20\%) among Japanese populations (Okano et al. 1994b). This mutation results in negligible enzymatic activity. We also confirmed a correlation between mutation genotype and the ability to convert phenylalanine to tyrosine by the phenylalanine loading test in the carriers of each mutation. The parents heterozygous for the R241C mutation formed more tyrosine than the parents carrying the $\mathrm{R} 243 \mathrm{Q}$ or $\mathrm{R} 413 \mathrm{P}$ mutation. It is therefore possible that the R241C mutation has much less effect on hepatic PAH activity in vivo. Coexpression analysis of these variant alleles in vitro may further explain the geneotype-phenotype relationship suggested in this study.

In European populations, the missense mutations A403V, V245A, E390G, and S87R have been associated with the non-PKU hyperphenylalaninemia phenotype (Guldberg et al. 1994). If other mutations with less effect on PAH activity are identified in the Japanese population, early clinical classification of infants with hyperphenylalaninemia will be much easier using PCR-based methods. The method may also be applicable to detect carriers to improve genetic counseling of non-PKU hyperphenylalaninemia families.

A lifelong dietary treatment was recommended even for those patients with serum phenylalanine levels between 400 and $600 \mu \mathrm{mol} / 1$, because untreated patients presented a lower IQ compared with treated patients at the age of 4 years (Costello et al. 1994). However, a recent investigation has indicated that adolescent and adult patients with untreated non-PKU hyperphenylalaninemia seem not to be at risk of intellectual and neurologic deficits (Weglage et al. 1997). As a consequence of these investigations, the dietary policy for non-PKU hyperphenylalaninemia is still controversial. Since the incidence of this disorder is extremely low in Japan $(1$ in 328,880$)$, sufficient data are not available on the outcome of the patients to determine the necessity of a restricted diet. Some patients have not been followed up clinically throughout childhood. Continuing investigations on the pathogenesis of non-PKU hyperphenylalaninemia using both biochemical and DNA-based methods are necessary.

Acknowledgment This work was supported by the research grant 8A10 for Nervous and Mental Disorders from the Ministry of Health and Welfare of Japan.

\section{References}

Aoki K, Wada Y (1988) Outcome of the patients detected by newborn screening in Japan. Acta Paediatr Jpn 30: 429-434

Costello PM, Beasley MG, Tillotson SL, Smith I (1994) Intelligence in mild atypical phenylketonuria. Eur J Pediatr 153: 260-263

Daiger SP, Reed L, Huang S-Z, Zeng Y-T, Wang T, Lo WHY, Okano Y, Hase Y, Fukuda Y, Oura T, Tada K, Woo SLC (1989) Polymorphic DNA haplotypes at the phenylalanine hydroxylase locus in Asian families with phenylketonuria (PKU). Am J Hum Genet 45: 319-324

Economou-Petersen E, Henriksen KF, Guldberg P, Güttler F (1992) Molecular basis for nonphenylketonuria hyperphenylalaninemia. Genomics 14: 1-5

Guldberg P, Henriksen KF, Thony B, Blau N, Güttler F (1994) Molecular heterogeneity of nonphenylketonuria hyperphenylalaninemia in 25 Danish patients. Genomics 21: 453-455

Gustafson S, Proper JA, Bowie EJW, Sommer SS (1987) Parameters affecting the yield of DNA from human blood. Anal Biochem 165: 294-299

Güttler F (1980) Hyperphenylalaninemia: Diagnosis and classification of the various types of phenylalanine hydroxylase deficiency in childhood. Acta Paediatr Scand Suppl 280: 1-80

Levy HL, Shie VE, Karolkewicz V, French WA, Carr JR, Cass V (1971) Persistent mild hyperphenylalaninemia in the untreated state. A prospective study. N Engl J Med 285: 424-429

Lin CH, Hsiao KJ, Tsai TF, Chao HK, Su TS (1992) Identification of a missense phenylketonuria mutation at codon 408 in Chinese. Hum Genet 89: 593-596

Okano Y, Eisensmith RC, Güttler F, Lichter-Konecki U, Konecki DS, Trefz FK, Tasovich M, Wang T, Henriksen K, Lou H, Woo SLC (1991) Molecular basis of phenotypic heterogeneity in phenylketonuria. N Engl J Med 324: 1232-1238

Okano Y, Hase Y, Lee DH, Takada G, Shigematsu Y, Ohura T, Isshiki G (1994a) Molecular and population genetics of phenylketonuria in Orientals: Correlation between phenotype and genotype. J Inher Metab Dis 17: 156-159

Okano Y, Hase Y, Shintaku H, Araki K, Furuyama J, Ohura T, Isshiki G (1994b) Molecular characterization of phenylketonuric mutations in Japanese by analysis of phenylalanine hydroxylase mRNA from lymphoblasts. Hum Mol Genet 3: 659-660

Takarada Y, Yamashita K, Kagawa S, Zhang QZ, Matsuoka A (1994) Genetic diagnosis of phenylketonuria III. Mutations of phenylketonuria hydroxylase gene in Orientals. Jpn J Clin Pathol 42: $1158-1164$

Wang T, Okano Y, Eisensmith R, Huang S-Z, Zeng Y-T, Lo WHY, Woo SLC (1989) Molecular genetics of phenylketonuria in Orientals: linkage disequilibrium between a termination mutation and haplotype 4 of the phenylalanine hydroxylase gene. Am J Hum Genet 45: 675-680

Wang T, Okano Y, Eisensmith RC, Harvey ML, Lo WHY, Huang S-Z, Zeng Y-T, Yuan L-F, Furuyama J, Oura T, Sommer SS, Woo SLC (1991a) Founder effect of a prevalent mutation in the Oriental population. Proc Natl Acad Sci USA 88: 21462150

Wang T, Okano Y, Eisensmith RC, Lo WHY, Huang S-Z, Zeng Y-T, Woo SLC (1991b) Identification of a novel phenylketonuria mutation in the Chinese: further evidence for multiple origins of PKU in Asia. Am J Hum Genet 48: 628-630

Wang T, Okano Y, Eisensmith RC, Lo WHY, Huang S-Z, Zeng Y-T, Yuan L-F, Liu S-R, Woo SLC (1991c) Missense mutations prevalent in Orientals with phenylketonuria: molecular characterization and clinical implications. Genomics 10: 449456 
Wang T, Okano Y, Eisensmith RC, Lo WHY, Huang S-Z, Zeng Y-T, Weglage J, Ullrich K, Pietsch M, Funders B, Güttler F, Harms E (1997) Yuan L-F, Liu S-R, Woo SLC (1992) Identification of three novel PKU mutations among Chinese: evidence for recombination or recurrent mutation at the PAH locus. Genomics 13: 230-231

Intellectual, neurologic, and neuropsychologic outcome in untreated subjects with nonphenylketonuria hyperphenylalaninemia. Pediatr Res 42: 378-384 